\title{
Academic Leadership Development Programs: A model for sustained institutional change
}

\author{
Ortrun Zuber-Skerritt and Ina Louw
}

\begin{abstract}
Purpose

This paper evaluates a leadership development program (LDP) for senior academic staff on 'qualitative research' after two years to establish the success, limitations and overall impact of the program in terms of personal, professional and organizational benefits.
\end{abstract}

\section{Design/methodology/approach}

We present the background, outline of the LDP and evaluation of results through participant feedback (1) at the end of the program; and (2) after two years, using a 'participatory action learning and action research' (PALAR) approach.

\section{Findings}

All participants were very positive about the design, conduct and learning outcomes of the program in terms of their own and their students' learning during and after the program. But although the workshop had prepared them, some had not cascaded their learning and skills by conducting similar programs in their department, faculty or university wide, which was one of the main original objectives to achieve a multiplier effect across the institution. We discuss various reasons for this shortcoming and develop a process model for positive institutional change management in higher education.

\section{Research limitations/implications}

Medium- and long-term effects of an LDP need to be followed up after a timespan of one to three or five years to establish whether the development has been effective and sustainable and to learn from limitations and shortcomings for future R\&D activities.

\section{Practical implications}

We identify the limitations and suggest practical institutional changes that encourage cascading of learning in theory and practice with a multiplier effect.

\section{Original/value}

Our process model for leadership development in higher education can be adopted, adapted or further developed by other scholars interested in designing, conducting and evaluating a sustainable LDP in their field and organization. 


\section{Introduction}

Universities and other higher education institutions across the world have for several decades recognized the value of developing leadership capacity among academic staff members. Leadership development programs (LDPs) for academics are usually used to cultivate the capacity of senior staff to promote and support the potential of their colleagues for contributing to creation and dissemination of knowledge through research, publication and teaching, and for other valuable knowledge-related contributions to their community and beyond. LDPs therefore can reward higher education institutions and society at large, as well as individual academics professionally and personally. Most universities offer academic LDPs in-house and/or off-campus, usually tailored to meet specific academic skills, needs or research fields. Because of the investment potential of these programs, it is important that they are run most effectively to maximize their benefits.

This article therefore explores the strengths and weaknesses of an academic LDP and offers a model for use within higher education institutions to guide success overall. The model offers guidance on design and implementation of academic LDPs for sustained institutional advancement and individual and collective academic development through capable academic leadership. We case study an intensive academic staff development program conducted in 2011 in a South African university on qualitative research methods and thesis writing for senior academics as leaders. This case study builds upon the studies of many professional development programs for academics conducted in South African higher education since 1995, including Speedy (2003), Fletcher and Zuber-Skerritt (2008); Fletcher et al. (2010) and Wood and Zuber-Skerritt (2013). This article and the LDP it case studies respond to Garcia and Gluesing's (2013: 423) call in this journal for researchers to use qualitative research methodology more extensively in organizational change programs "to help uncover new organizational phenomena; build and test theories of change; and create new methods that researchers can use specifically in international change studies."

The main shortcoming of most academic staff development programs in higher education, including LDPs, appears to be the limits on their capacity to achieve sustainability. Individual participants may gain benefits for their own research proficiency and their supervision of postgraduate students immediately after the program, but the institution as a whole is deprived of the flow-on benefit when these individuals stop using their newly acquired knowledge and skills or they leave the institution. A significant problem - one that is poorly appreciated, often not even recognized, and to some extent is intrinsic to the unsustainability shortcoming - is methodology. LDPs are usually based on the traditional model of instructional design and theory/content orientation, rather than on learning and research processes informed by models of experiential, lifelong action learning that foster sustainable outcomes.

We have shown that participatory action learning and action research (PALAR) can be an effective methodology for sustainable professional and leadership development (Louw \& Zuber-Skerritt, 2009; Wood \& Zuber-Skerritt, 2013; Zuber-Skerritt, 1992a,b). The purposes of the present paper are therefore to identify how and why the intended outcomes of LDPs are not always sustained and on that basis to offer a model 
for academic LDPs with understandings and processes for sustainable positive outcomes. The LDP in our case study used PALAR as a methodology and sustainability as one of its objectives. A condition for participation was the participants' agreement and willingness to conduct qualitative research (QR) workshops themselves in their department, faculty or university wide after the program and to cascade their learning and skills to colleagues and postgraduate students in the social sciences by 'training the trainers', to achieve an enduring multiplier effect in this university.

The process model of academic leadership development that we present in this paper draws from our experiences with PALAR for professional development programs in universities in various countries. This model aims at avoiding shortcomings through the active participation of academics throughout the program and beyond. Particularly through the cascade effect of program participants passing on to colleagues what they have learned in the program, it works to ensure sustainable development at the institutional level as well as for individual academic staff. A further strength of this model is flexibility. It can be adopted, adapted or further developed by academics and others who are interested in designing, conducting and evaluating a sustainable LDP in their field and/or organization.

For evaluating this LDP we have used the quality criteria developed by Fletcher and Zuber-Skerritt (2008: 88):

1. The program facilitator's expertise with regard to credibility and international reputation, in-depth knowledge in the field, facilitation and process management skills, and the quality of the program materials;

2. The facilitator's use of adaptive planning after a needs analysis at the beginning of the program and after each module or session, using:

3. Responsive evaluation based on constant feedback from participants to enable the facilitator - and participants if needed - to act on this feedback immediately;

4. Critical events that contribute to participants' transformational learning and understanding of the new research paradigm;

5. Application, i.e., cultivating participants' ability to recognize ways to apply the program's content to their own professional and private lives; and

6. Self-efficacy, enthusiasm and confidence of participants in applying their learning from the program continuously in their professional life, which includes cascading their learning to colleagues after the program.

As mentioned earlier, our criteria also included sustainability, through participants' ability and willingness to cascade their learning, knowledge and skills to others in the institution after the program. Heightened consciousness of the need for sustainability in our world in the $21^{\text {st }}$ century has deepened our appreciation of PALAR's utility to contribute to sustainable outcomes through the cascaded learning it entails.

We used Meynell's (2005) second-order evaluation methodology, including semistructured interviews, 'scheduled conversations' (such as pre-arranged informal meetings and phone or e-mail conversations), informative 'chance conversations' with members and associates of the university, program attendance and participant observation, 'first-person' reflections in a learning journal, and listening to 
participants' stories and their reflections on their experiences. We recognize these second-order qualitative research methods as better suited to a PALAR approach because they are more in keeping with the basic philosophical assumptions of our R\&D strategy than first-order quantitative research methods. The main aims of our evaluation strategy are improved academic practice (the action part) and the participants' understanding, learning, insights and new knowledge, as 'practical theory' related to their workplace in higher education (the research part).

The first author designed and facilitated the LDP case studied in this paper. The second author was a participant observer in the LDP in 2011 and interviewer for evaluation of it in 2013. We collaborated in thinking, writing and reviewing this paper. Together we reflected on our analysis and interpretations, discussed our conclusions, and finally validated our research by participant confirmation or member check. We allowed a two-year interim between conduct and evaluation of the program given the cascading process required of participants after the program and the sustainability it aimed to achieve.

First, we briefly describe the background of the case study in the South African context. We then outline the aims and activities of the LDP and its outcomes on the basis of participants' feedback during and at the end of the program and through individual interviews two years later. After discussing the results we present the model of leadership development in higher education that we have developed in the light of the case study experience and the literature, before drawing our conclusions.

\section{Background}

South Africa's higher education landscape has changed rapidly over the past few years in moves to address national inequalities post-apartheid (Olivier, 2007: 1128). Yet inequalities and dysfunction still disfigure the higher education system (CHE, 2013: 54). Despite efforts to increase the number of postgraduate students, particularly black students, most universities have been unable to fill all their postgraduate openings (Mouton, 2007: 1088). The problem is even greater at universities of technology (UoTs). Research was not one of their core functions until they were transformed from 'technikons' into universities over roughly the past decade. ${ }^{1}$ The new 'academics' could not become proficient supervisors overnight, leaving many inexperienced and overwhelmed while trying to take on the new academic supervisor role (Mouton, 2007: 1090). Furthermore, many in the current cohort of university researchers are approaching retirement (Mouton, 2007: 1079). These circumstances have made it imperative for UoTs to plan and implement academic staff development programs to empower and enable their academic staff to be effective supervisors and solid researchers (Nsimande, 2007: 1119).

At Tshwane University of Technology (TUT) - the locus of our case study institutional programs address generic research skills and postgraduate supervision skills. The program coordinator invited the first author to conduct a 'Master Class' on

\footnotetext{
1 The uniquely South African designation of 'technikon' was abandoned with conversion of these institutions into 'universities of technology' (McKenna \& Powell, 2009).
} 
'Qualitative Research' in 2011 with senior academics interested in qualitative research. Although - in fact, because - many of them had experience in qualitative research, the aim was to equip them with both a model and capacity to present similar workshops themselves to reach larger groups of academic colleagues, beyond the narrow reach of their own students.

Not only in the South African context, but also in most countries worldwide, the dominant paradigm in higher education is shifting from the traditional positivist paradigm using mainly quantitative research methods to the emerging non-positivist paradigm using predominantly qualitative research methods. Social scientists are acting on recognition that just as human and social problems are different from problems in the natural sciences, so too are the research approaches most appropriate for addressing these problems. Human behaviour cannot be predicted and 'measured' exactly as non-organic, chemical or physical reactions can be. So many aspects of human behaviour reveal their characteristics through their quality rather than their quantity. Human constructs, opinions, views and values differ from individual to individual and usually change over time. They need to be elicited and 'evaluated' by qualitative methods such as open-ended questions, interviews, dialogue, reflection diaries, focus groups and other small-group discussion, to obtain richer data and deeper understanding of a complex situation than quantified research could generate. Exceptions include large-scale surveys using statistics to identify national trends in human or social behaviour.

Social scientists have also argued that the human and social sciences require methodologies different from the natural sciences because the nature, behaviour and mind of humankind constitute a complex whole, which cannot be observed objectively or in parts by outside researchers. Many have recognized that observations are not neutral, objective or value free but are subjective interpretations shaped by the observer's value system, ways of viewing the world, and so their theoretical framework - right down to what the researcher chooses or accepts as the topic of their analysis and the particular questions they ask about it. This is why 'subjects' need to be 'participants' in the research process (e.g., in analyzing and interpreting data) so that the ineluctable subjectivity of research is recognized and appreciated for how it has shaped the research, to enable more informative findings and creation of more accurate knowledge. Participatory action learning and action research (PALAR), used in this LDP, is an affective-socio-cognitive process that results in transformational professional learning and is confirmed by neuroscientific research. Zuber-Skerritt, Fletcher and Kearney (2014, in press) conclude: “... the PALAR paradigm ... appreciates the validity of differing values and world views since they are informed by the distinctive experience and life journeys of those who hold them." This means, participant researchers are not only cognitive thinkers, but also recognize and critically reflect on their feelings and social relationships. Learning from and with one another and engaging in critical dialogue promote collective critical interpretation of meaning in an organization. Through the reflexive and critical communication people are able to learn, lead and create knowledge and to develop cultural competences necessary for establishing a civic attitude and sustainable organizational change (Book and Philips (2013).

A shift in the dominant paradigm of research in the social sciences is evident in growth of this literature at an exponential speed in the last two decades, especially on 
grounded theory (Clarke \& Charmaz, 2013; Corbin \& Strauss, 2013; Dey, 1999), systems theory (Checkland \& Poulter, 2006; Burns, 2007), critical theory (Hunter et al. 2013; Kemmis, 2008), action theory (Argyris \& Schön, 1974; 1996; Zuber-Skerritt, 2011) and experiential learning theory (Kolb, 1984; Zuber-Skerritt \& Teare, 2013). Monographs, handbooks and students' guides on qualitative research methods based on these theories and philosophical assumptions have mushroomed in recent years, for example: Holliday (2002), Ten Have (2004), Marshall and Rossman (2006), Silverman (2007; 2013), Somekh and Lewin (2005; 2011), Steinar and Brinkmann (2008), Denzin (2009), Denzin and Lincoln (2011), Lichtman (2013), Ritchie et al. (2013), Barbour (2013), Yin (2013), Sikes (2013) and Gray (2013).

Qualitative research methodology in the emerging paradigm of non-positivist research, especially 'participatory action learning and action research' (PALAR) and 'action leadership' (Zuber-Skerritt, 2011), has proven to be effective in change management programs, whether in higher education, management education or organization development generally. The LDP in the case study discussed below focuses on qualitative research methods in the social sciences to equip senior academics in a university of technology to become leaders in their field of research and to cascade their knowledge and skills to their colleagues and postgraduate students in the social sciences.

\section{Case Study}

\section{Outline of the LDP}

The aims of this LDP were to develop among participants high level, practical and effective research skills for data collection, analysis, interpretation, and writing academic papers, dissertations and theses involving qualitative research.

The philosophy, methodology, process, structure and content of this kind of professional development program in higher education are described in detail in Zuber-Skerritt (2009: 139-166). Here we provide a brief executive summary. This academic LDP was designed and aimed to:

- Provide an overview of paradigms; types of knowledge; action research and qualitative research methods, techniques and tools; main problem areas in postgraduate research, and strategies to avoid or overcome these problems;

- Identify and meet participants' expectations, needs and priorities concerning qualitative research;

- Be based on adult learning theories/principles and used processes of experiential learning for lifelong learning, action learning and action research;

- Focus on individual (and team) project design, including the research question/problem, significance of the work, methods used and justified, timeline, and original contribution to knowledge; and

- Provide practical advice on writing proposals, literature reviews, and preparing/publishing qualitative research reports, papers, dissertations and theses. 
Skills/competencies for participants to acquire through attending the LDP included:

- How to design a qualitative research project;

- When to use quantitative and qualitative research methods (differences and similarities; tips on effective use and how to avoid pitfalls);

- Academic writing involving qualitative research; and

- How to structure a thesis, dissertation, article or book and to write for a particular readership.

Outcomes for participants on completing the program included:

- Ability to make informed choices concerning epistemology, ontology, research paradigms and methodologies (e.g., grounded theory, action research, case study methodology);

- Knowledge of a wide range of qualitative research methods and techniques; ability to independently acquire knowledge of a new method, to teach it, and to adapt it to a particular purpose;

- Confidence and competence to conduct independent qualitative research;

- Time management ability for completing research and submitting a thesis/ manuscript on time; and

- Appreciation of collaboration, sharing ideas and forming networks and support groups (e.g., for publication).

\section{Assessment criteria:}

Participants' ability to acquire, understand and teach a qualitative research method was assessed by the course presenter and peers, using criteria related to the following areas:

- Content;

- Clarity;

- Structure;

- Explanation of method and its applications;

- Answering questions after presentation;

- Giving examples in presentation and in question time; and

- Time management.

\section{Selection of participants}

The participants were nominated by the deans of the faculties and by he directors of HEDS (Higher Education Development and Support). Due to the intensive nature of the program, the maximum number of participants was restricted to 12 . On day one, only 11 people arrived and after day one, two withdrew for personal reasons, resulting in only nine participants undertaking and completing the program.

\section{Results in 2011}

In 2011 the nine participants (P) in the LDP showed great interest in the topic of qualitative research throughout the three days. They engaged actively in all aspects of the program and appreciated its participatory, action-oriented approach to self-directed 
learning and research, encouraging reflection, debate, small-group discussion, networking and a "new way of thinking" (P6). This LDP, in literally practising what it preaches, provided an excellent model for participants to use in their own efforts to cascade their learning to colleagues through interactive, practical programs. Here we overview participants' stories using verbatim quotations from the feedback forms they completed at the end of the program.

Highlights they reported were the "collaboration between people in different faculties, the collaborative nature of the workshops and the [second] day of independent study and reading" (P1). For the independent study, each participant had to select one qualitative research method, and research, reflect and prepare a talk on it to present the next day, orally in ten minutes and in concise written form (up to two pages). Whilst they found it a daunting task at first, through their collaboration with each other and active consultation with the first author as facilitator, fuelled with a healthy dose of enthusiasm, all participants performed well. Their peer assessment forms where they noted 'positive' and 'negative' aspects of the presentations and what they found 'most interesting' indicate both their understanding of the qualitative research method they had studied and their capacity to cascade their learning about it to others through their presentation. The qualitative research methods they explored and presented were ethnography, case study, phenomenological inquiry, discourse analysis, qualitative content analysis, nominal group technique and interviews (general overview and individual interviews).

Participants were enthusiastic about the "opportunity to get a good grounding in the QR approach" (P2) by learning new qualitative methods that they could use in their own research and teaching their students. They appreciated the "networking opportunities with other senior researchers" (P3) and the "team work that strengthened ties with fellow qualitative researchers" (P4). They also commented on the facilitator's "deep knowledge" (P3), "quality inputs" (P4), "positive way of presenting. All the references we got - no excuse for not reading!" (P6) and "communication skills - she sought consensus whenever something had to change in the program. Her experience in activities shined through the advice she gave us" (P5).

On the whole, participants reported that they felt well equipped and confident to facilitate similar programs or workshops to their colleagues and students. They considered the LDP "practical, participatory in nature - making it more exciting" (P8), "very time effective - lots of work covered in three days" (P6) and "with exceptional learning - really walking out here with lots of knowledge, ideas and also a plan" (P9). Another participant commented on his/her "own action plan to improve my own practice. I think we have the potential to stay together. [We can take] future actions to improve postgraduate output in the whole university" (P7).

Most participants made no real negative comments and no or few suggestions for improvement. Three people would have liked more time and three others mentioned the venue: "It was held on campus and your office work distracts you. Have the workshop off campus" (P7). "Personally I struggled to keep the focus because I was in my office and normal day work interfered" (P9). "The venue is not suitable for collaborative, interactive presentation technique, but she [the facilitator] made good use of what was available" (P6). Another critical comment was the "lack of commitment from some individuals" (P1) referring to the three people who enrolled 
but did not participate, with the suggestion for improvement that the university should have a "contract with participants expecting them to carry the financial burden if they withdraw from a workshop" (P1).

\section{Results in 2013}

During 2011 the feedback was anonymous and the numbers assigned to participants do not correspond with the numbers assigned to the interview participants in 2013. Data collected in 2013, through individual, semi-structured interviews, focused on four questions relating to the LDP in 2011:

1) What stands out for you after two years?

2) What is the biggest gain you have experienced?

3) How have you used the knowledge from this LDP?

4) In hindsight, do you think the LDP could be changed to improve its effectiveness?

Most of the nine participants had experienced a significant change in their working profile in the two years since the LDP. One resigned due to relocation with her husband, three were promoted to (or are acting in) senior positions involving more administrative work, one person is back into a full-time teaching position after acting in a managerial position, and two have been seconded to non-teaching positions. Two participants did not respond to our invitation for an interview, one having left TUT since the LDP. Here is a brief summary of the seven interviewees' responses to our questions in the 2013 interviews.

What stands out for you after two years?

The participants' reactions were strongly positive. Most could still vividly remember the program and were clear on what stood out for them in hindsight. Most spoke of the presenter and her presentation style, e.g., "Her way of conducting the sessions was great" (P1). The participants appreciated her style, her communication and negotiation around their needs, her knowledge and the format of the workshop, e.g., "the presenter was so knowledgeable" (P4).

Some participants remembered the group of people who attended the LDP, e.g., "the enthusiasm in the group was so exciting" (P2) and "I felt honoured to be included in a class where professors and deans were included" and "the fact that all the participants worked and presented was impressive" (P7). Many participants also recalled their emotions during and shortly after the program: 'enthusiasm', 'confidence', 'positive feelings' and being 'empowered'; e.g., "I left with a wow" (P2) and "I left positive about qualitative research as I learned so much" (P5).

\section{What is the biggest gain you have experienced?}

For most participants this was acquiring knowledge. Most indicated their biggest gain was knowledge acquired during the program. Some identified practical knowledge; e.g., "... the use of a reflection diary and all the different methods were useful to me" (P6). Some identified a new approach to learning - from and with others, e.g. "It was useful to present and listen to others and evaluate their presentations. It made me comfortable" (P1). Also "I learned from the presenter and the group. I got to know 
other active researchers and my students are benefitting" (P5). Some also spoke of acquiring knowledge from the resource documents presented to all participants at the start of the program; e.g., "I have used the resource material with my students and as an author I've used it as an immediate resource and as reference material" (P2).

Confidence was clearly another valuable gain. Most explained or implied that increasing their knowledge had increased their confidence; e.g., "when I share now, I share with confidence" (P4). For P5, “... in conversations [about research] I have more knowledge and feel more confident to share my opinion"; "I was confident to use content analysis with some of my postgraduate students".

Some participants associated their biggest gain with the quality of the presenter being able to work with her in the program, and from their individual coaching sessions with her (30 minutes each), conducted throughout the (second) independent study/ preparation day; e.g., "The personal coaching was the best. She advised me on how to use my daily work to collect data and possibly publish it" (P1). Another observed, "My approach with my students has changed. I am not only asking them about their work, I also discuss their learning journey [as a result of the coaching I received]" (P5). The same participant mentioned "the presenter - her whole research history and wealth of knowledge".

\section{How have you used the knowledge from this $L D P$ ?}

The interviewer asked the participants what they had done with the knowledge they acquired during this program. We have grouped the answers loosely in four categories, as: a person/a researcher, supervisor/a researcher, presenter/ and as author. Some respondents mentioned personal application; e.g., "I am a more holistic researcher now" (P5), and "I have confidence when mentoring some of my colleagues" (P7). Others also pointed to their supervisory roles; e.g., "I encourage my students to use it too [reflection diary]" (P6). One responded: "Depending on the topic, I allow my students to only write a summary of the interview data and not transcribe it verbatim. It helps the students a lot" (P3). Many use the resource material continuously; e.g., "I use many resources and they have become part of me" (P3); and "... as an author I have used it as an immediate resource and as reference material" (P2).

Four of the participants had presented workshops after the LDP to cascade their learning to colleagues and others within the university and beyond. Their responses identify different and similar ways in which they have used their knowledge from and through the program. One participant explained how she had drawn comprehensively from her program experiences to present within her department: "I adapted the facilitator's style from this program to fit the format of the workshops I had to present and I am busy finding my own unique style. I keep it interactive and during the second workshop I also allowed time for presentations and evaluation by peers and the participants found it very useful. I received many compliments afterwards from the participants and that is very rewarding for me" (P7). Another observed similarly about presenting in her department "I realized again that a presenter must keep the program as practical as possible ... not lecturing; they [participants] must be doing. Talk only is useless" (P3).

One participant presented a short, 'condensed' session in a faculty forum for colleagues preparing their postgraduate research proposals. She drew from the essence 
of the program to share with them (P2). Another participant had taken their learning outside the university, advising "I was asked by a local government to assist them and I encouraged them to do qualitative research [in their work] and I could share with confidence" (P4).

What could be changed to improve the effectiveness of the program?

The 2013 data presented only two suggestions. One concerned the need to create ways to continue networking among and beyond participants after the LDP, since the participants had not sustained the network and relationships developed during the program in the longer term. The second suggested more attention to data analysis, e.g., "Atlas.ti [a software analysis package] could be added" (P6). We believe these two suggestions marry well. The participants can be further encouraged to take responsibility for sustaining and expanding their LDP network after the program to ensure quality and longevity of outcomes - for the institution and for the individuals involved as participants in the program, in the cascaded learning, in research projects, or through other outlets such as publications. The networks can usefully maintain ongoing discussion and update members on computer programs for data analysis, which will not only help to cascade knowledge and promote sustainability but also serve to reinforce the linkages between network members. In the next section we discuss our own suggestions for improvement.

\section{Discussion of Results and Recommendations}

In evaluating the program after two years in light of our interviews with most of the participants, we identify two aspects that can be modified to improve the quality and sustainability of program outcomes for both the institution and people involved. One is the shortcoming in conducting the networking and continued engagement that participants planned and agreed to at the end of the LDP. The group was sufficiently dynamic and cohesive to form a strong collaboration at the time of the LDP as their behaviour during the program demonstrated and comments such as, "I think we have the potential to stay together" (P7) suggest. Yet as a participant said in 2013 "we didn't pull it off" (P2). Had they "pulled it off", their efforts would have been strategically very valuable for the university and for its staff/student community. Their aspiration to create a postgraduate support group was noble, but without continued cohesion among the participants it was never established. Participants offered various reasons concerned with time and priorities; e.g., "everybody got busy" (P2), "we all had very different thoughts about it [collaboration network] and it just stopped" (P3). With participants from different campuses, “... logistically regular meetings will be a problem" (P7). One participant expressed the need for a leader/facilitator: "This group needed a 'driver' - like everything in life" (P3).

The second downside of the program is inability to maximize and embed benefits for the university and its staff and students through the multiplier effect. The LDP was designed to empower a selected group of senior academics to cascade their knowledge to other groups of researchers in the university. Most participants again gave reasons concerned with time and priorities, particularly their heavy workload, for not cascading their knowledge across the university's learning community. The reasons mentioned include being promoted to a more administration-intensive position and acquiring more committee responsibilities. However we identify a deeper source of the problem in the absence of a wider program design, where institutional leaders have a 
comprehensive plan with strategies for before and after the three-day program to maximize, embed and sustain professional learning and other positive outcomes through leadership development and cascaded learning.

Conducting the program on campus detracted from the LDP's constructive capacity since participants could not fully engage and focus with workaday distractions literally so close at hand. That is why we recommend that these LDPs should be offered as residential programs. This inevitably increases costs, but our experience makes clear that it is a wise investment. As mentioned earlier, university authorities sent the invitation to attend the LDP to all deans to nominate delegates. The participatory nature of the program required that we cap the number of participants at 12. Although there was a waiting list as more than 12 people were nominated, the three initial participants who signed on but then withdrew from the program prevented others from attending. As one of the LDP participants suggested, we recommend that universities enter into an agreement with staff who receive professional development opportunities that are costly for the university. Failure to complete the program should result in the participants being responsible for the financial cost of their expected participation in the program.

The 2013 data reveal participants' insufficient attempts to cascade knowledge within the university due to their heavy workloads. We recommend that universities ensure that participants who are expected to cascade their knowledge are given time and opportunity to do so, e.g., by including the cascading activities in their Key Performance Areas (KPAs). That way the university can ensure fuller benefits from the multiplier effect. Progress can then be monitored, and development more solidly institutionalized to make overall benefits from the LDP more sustainable across the university and its community.

Some participants expressed their disappointment that they could not continue their collaboration with each other after the LDP. Generally, the literature of Psychology informs us that a leaderless group should not exceed six members. With more than six members, a group needs a chairperson or leader/facilitator to work effectively together. On this basis we recommend that groups should be limited to five or maximum six members so that together they can achieve their common goal. Where numbers warrant it, participants should form more than one group.

Continued collaboration and networking would have been beneficial for the participants and their colleagues and students, to continue and sustain the learning and academic leadership and other professional development that participants undertook through the LDP. The continuity would have been highly beneficial for the university as well. As an intensive staff development program in qualitative research methods and thesis writing for senior academics as leaders, this LDP was designed to foster the strong research culture that UoTs need but are usually not well positioned to develop. This university of technology, as a former technikon, very much needs to implant and grow the research culture that the LDP sought to embed. Its institutional status to some extent depends upon building its research profile. These observations are true for all universities that were previously some type of college such as for technology, teaching and so forth. We are therefore considering how an LDP can not just prepare but also encourage or even require participants to embed understandings and processes of qualitative research to foster a research culture within the university after they have 
completed the LDP. We expect this will entail PALAR within and beyond the cascaded learning processes, consistent with the methodology of the LDP.

In the next section we consider how to achieve effective change in organizations, including higher education institutions, through LDPs. We also consider why many so-called 'change' programs do not produce transformational or sustainable change. We present a model for leadership development that we have designed on the basis of our experience in LDP in higher education, as a guide for programs that achieve sustainable development and to inspire further thought about this important developmental task in any institution or organization.

\section{A Model of Leadership Development}

Beer et al. (1990) maintain that three major interrelated factors are needed to revitalize an organization: Coordination or teamwork, a high level of commitment that is necessary for coordinated action, and competencies for problem solving as a team, including analytical and interpersonal skills.

The participants in our LDP perceived on completing the program that they had achieved a high level of teamwork, commitment and competencies in qualitative research and problem solving. But as we appraise the outcomes, an ingredient vital to sustaining the changes from personal and professional levels to a systems approach to institutional change was missing. That ingredient is an institutional system. Beer et al. (1990: 173) conclude from their research that organizations

... need a particular mind-set for managing change: one that emphasizes process over specific content, recognizes organization change as a unit-by-unit learning process rather than a series of programs, and acknowledges the payoffs that result from persistence over a long period of time as opposed to quick fixes. This mind-set is difficult to maintain in an environment that presses for quarterly earnings, but we believe it is the only approach that will bring about successful renewal.

Zuber-Skerritt (2011: 172-177) has developed a model of action research for organizational change, adapting the linear six-step model of managerial interventions for organizational change prepared by Beer et al. (1990). Her adapted circular model shows the overlapping theories of action research and force field theory with the model of task alignment by Beer and his associates. Here we adapt this model further into another new eight-phase circular task alignment model in combination with the four phases of participatory action learning and action research (PALAR) for sustainable institutional change and development, as shown in Figure 1. 


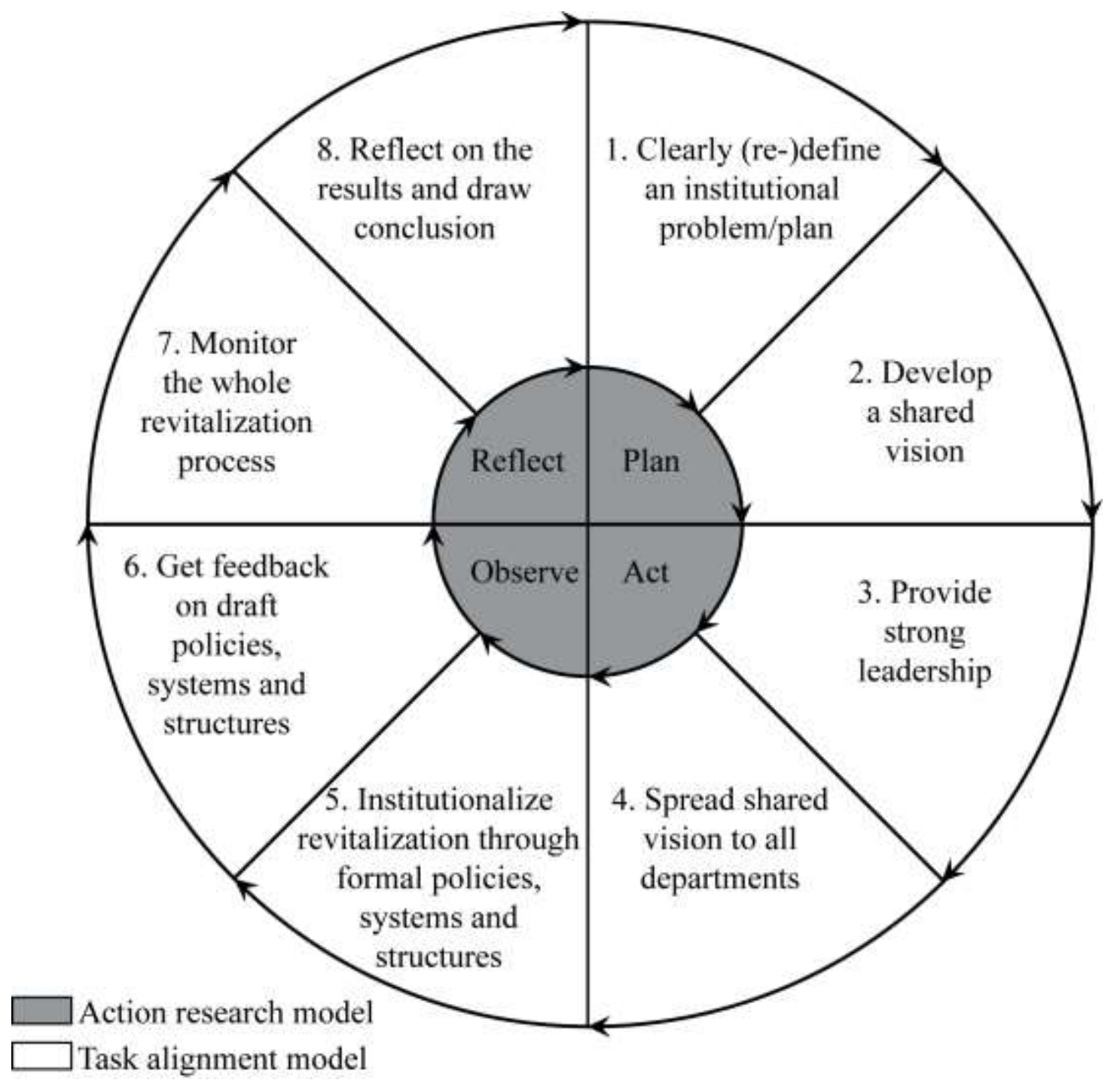

Figure 1: Model of institutional change using participatory action learning and action research (PALAR)

(1) We start with a clearly defined institutional problem and plan; (2) then develop a shared vision and create consensus among stakeholders; (3) provide strong leadership in supporting teams and replacing dysfunctional staff if necessary; (4) spread the new, shared vision to all departments that work out their own applications in their particular contexts; (5) institutionalize the revitalization through formal policies, such as key performance indicators, systems and structures for sustainability and reward for success and persistency; (6) get feedback on draft policies, systems and structures from all stakeholders; (7) then monitor the whole revitalization process using ongoing evaluation and feedback from all departments; and finally, (8) reflect on the results of the evaluation and draw conclusions on what worked well and what did not and why or why not, for informing the next cycle(s) of PALAR when re-defining institutional problems and plans.

This cyclical model of sustainable institutional change may be logical and familiar to most action researchers in the non-positivist paradigm. An exception here may be phase three: that no organizational system can work well and be sustainable unless dysfunctional staff are replaced by willing, committed and capable staff. This is 
definitely challenging to some organizations, including most institutions of higher education, but it is not at all the practice in higher education in South Africa and several other countries. They will have to change quickly and radically, because if they do not, they will not survive in their national or international contexts and in global competition, as predicted and argued convincingly in a recent report published by the Institute for Public Policy Research (IPPR) in the UK (Barber et al., 2013) entitled An Avalanche is Coming: Higher Education and the Revolution Ahead. Any university that is reluctant or too slow to change and that stands still in the path of the avalanche will be swept away, because students will be able to access the best learning systems and courses globally by new technology.

\section{Conclusions}

Qualitative research makes a valuable contribution to the educational and social sciences. The international literature using this approach has mushroomed in the last two decades. Nevertheless, many researchers - educated in the traditional, positivist paradigm of research - lack a true understanding of qualitative research methods and an ability to use them in their research. Yet knowledge of qualitative research and its underlying philosophical and methodological assumptions is essential for high quality research and thus for the practical and intellectual knowledge outcomes, theses, publications and successful grant applications that it feeds. Research concerning people's views, values, strategies, behaviours, professional development, learning and transformation, and organizational and leadership development/change is more likely to yield rich data and most useful results if it is carried out through predominantly qualitative research methods, rather than through only quantitative/ statistical methods.

Our case study has shown that a group of senior academics in a South African university of technology after attending an intensive three-day LDP on qualitative research $(\mathrm{QR})$ reported they had gained knowledge and skills in $\mathrm{QR}$, as well as confidence to teach QR to their colleagues and students. Participants confirmed that all six quality criteria developed by Fletcher and Zuber-Skerritt (2008) had been met: (1) the facilitator's expertise; (2) her use of adaptive planning, meeting their needs; and (3) responsive evaluation based on their constant feedback; (4) critical events, such as resource materials, 'reflection diary', individual coaching sessions, and learning how to learn and how to teach QR methods (on independent study day); (5) application of program content to their own professional and private lives; and (6) their self-efficacy, motivation, enthusiasm and confidence in conducting QR workshops after the program.

However, our new criterion of sustainability had not been met to the extent we expected. The main reasons that participants identified for not sustaining their networking and collaboration were lack of time, high workload, promotion to senior administration positions or leaving the university. We suggest an additional reason: as with most universities, this UoT did not have in place an institutional system to ensure continued collaboration, cascading and sustainable development of QR to promote an active research culture across the university. In response we have developed a model of institutional change using participatory action learning and action research (PALAR). The four phases at the right side of the model were included to some extent in the design of the LDP: (1) a clear plan designed to develop (2) a shared vision amongst stakeholders and participants before the program, (3) providing strong 
leadership in QR through this group of senior academics, and (4) spreading the shared vision to all faculties represented by members of this group.

However, what was missing was the left side of this model: (5) an institutional system with (6) agreed policies and structures to (7) monitor and sustain the whole development process after the program. We have therefore added (8) reflection on the results of our case study and drawn the conclusion that universities need to become faster, more creative and more radical in their continuing change and systems thinking. They need to support the best and most committed staff, replace dysfunctional staff, and establish a system to penalize those whose irresponsible behaviour robs others of valuable learning opportunities. This may entail making them pay for accepting a place in a costly professional development program and then by not attending the program effectively stealing another person's opportunity to participate and contribute to the university's development and academic goals. These are but a few examples of the new mindset needed in the higher education sector in the $21^{\text {st }}$ century. As Barber et al. (2013) urge stridently, more research and development are needed here to escape the avalanche that is coming.

\section{References}

Argyris, C., \& Schön, D. A. 1974. Theory in Practice: Increasing Professional Effectiveness. San Francisco: Jossey-Bass.

Argyris, C., \& Schön, D. A. 1996. Organizational Learning II: Theory, Method and Practice. Reading, Mass.: Addison-Wesley.

Barber, M., Donnelly, K., \& Rizvi, S. 2013. An Avalanche is Coming: Higher Education and the Revolution Ahead. London: Institute for Public Policy Research. Available at: http://www.ippr.org/publication/55/10432/anavalanche-is-coming-higher-education-and-the-revolution-ahead.

Barbour, R. 2013. Introducing Qualitative Research: A Student's Guide to the Craft of Doing Qualitative Research (Second ed.). Thousand Oaks, Ca.: Sage.

Beer, M., Eisenstat, R. A., \& Spector, B. 1990. Why change programs don't produce change. Harvard Business Review (November - December), 158-166.

Book, L., \& Philips, D. P. (2013). Creativity and Entrepreneurship: Changing Currents in Education and Public Life. Cheltenham: Edward Elger.

Burns, D. 2007. Systemic Action Research: A Strategy for Whole System Change. Bristol, UK: Policy Press.

Checkland, P., \& Poulter, J. 2006. Learning for Action: A Short Definitive Account of Soft Systems Methodology and its Use for Practitioners, Teachers, and Students. Chichester: Wiley.

Clarke, A. E., \& Charmaz, K. (Eds). 2013. Grounded Theory and Situational Analysis (Volume Set of Four Books). London: Sage.

Corbin, J., \& Strauss, A. 2013 Basics of Qualitative Research: Techniques and Procedures for Developing Grounded Theory (Fourth ed.). Thousand Oakes, Ca.: Sage.

CHE - Council on Higher Education. 2013. A proposal for undergraduate curriculum reform in South Africa: The case for flexible curriculum structure. Pretoria: Council on Higher Education: Task team on undergraduate curriculum structure.

Denzin, N. 2009. Qualitative Inquiry Under Fire. Walnut Creek, Cal.: Left Coast Press. 
Denzin, N. K., \& Lincoln, Y. (Eds). 2011. Handbook of Qualitative Research (Fourth ed.). Thousand Oakes: Sage.

Dey, I. 1999. Grounding Grounded Theory: Guidelines for Qualitative Inquiry. San Diego: Academic Press.

Fletcher, M. A., \& Zuber-Skerritt, O. 2008. Professional development through action research: Case studies in South African higher education. Systemic Practice and Action Research, 21, 73-96.

Fletcher, M. A., Zuber-Skerritt, O., Albertyn, R. M., Bartlett, B., \& Kearney, J. 2010. Meta-action research on a leadership development program: A process model for life-long learning. Systemic Practice and Action Research, 23(6), 487-507.

Garcia, D., \& Gluesing, J. 2013. Qualitative research methods in international organizational change research. Journal of Organizational Change Management, 26(2), 423-444.

Gray, E. (Ed.). 2013. Doing Research in the Real World (Third ed.). London: Sage.

Holliday, A. 2002. Doing and Writing Qualitative Research. London: Sage Publications Ltd.

Hunter, L., Emerald, E., \& Martin, G. 2013. Participatory Activist Research in the Globalized World: Social Change Through the Cultural Professions. New York: Springer.

Kemmis, S. 2008. Critical theory and participatory action research. In P. Reason \& H. Bradbury (Eds), The Sage Handbook of Action Research: Participative Inquiry and Practice (pp. 121-138). London: Sage.

Kolb, D. 1984. Experiential Learning: Experience as the Source of Learning and Development. New Jersey: Prentice Hall.

Lichtman, M. 2013. Qualitative Research for the Social Sciences. London: Sage.

Louw, I., \& Zuber-Skerritt, O. 2009. Reflecting on a leadership development program: A case study in South African higher education. Perspectives in Education, 27(3), 237-246.

Marshall, C., \& Rossman, G. B. 2006. Designing Qualitative Research (Fourth ed.). London: Sage.

McKenna, S., \& Powell, P. (2009). Only a Name Change: The move from Technikon to University of Technology The Independent Journal of Teaching and Learning, 4, 37-48.

Meynell, F. (2005). A second-order approach to evaluating and facilitating organizational change. Action Research, 3(2), 211-231.

Mouton, J. 2007. Post-graduate studies in South Africa: Myths, misconceptions and challenges. South African Journal of Higher Education, 21(8), 1078-1090.

Nsimande, R. 2007. Using 'currere' to re-conceptualize and understand best practices for effective research supervision. South African Journal of Higher Education, 21(8): 1117-1125.

Olivier, M.A.J. 2007. Postgraduate supervision: For better or for worse. South African Journal of Higher Education, 21(8): 1126-1141.

Ritchie, J., Lewis, J., McNaughton Nicholls, C., \& Ormston, R. (Eds). 2013. Qualitative Research Practice: A Guide for Social Science Students and Researchers (Second ed.). London: Sage.

Sikes, P. (Ed.). 2013. Autoethnography (Volume Set of Four Books). London: Sage.

Silverman, D. 2013. Doing Qualitative Research: A Practical Handbook (Fourth ed.). London: Sage.

Silverman, D. (Ed.). 2007. Qualitative Research: Theory, Method and Practice (Second ed.). London: Sage. 
Somekh, B., \& Lewin, K. (Eds). 2005. Research Methods in the Social Sciences : A Guide for Students and Researchers. London: Sage.

Somekh, B., \& Lewin, C. (Eds). 2011. Theory and Methods in Social Research (Second ed.). London: Sage.

Speedy, S. (Ed.). 2003. Women Using Action Learning and Action Research: The South African Context. Lismore (Australia): Southern Cross University Press.

Steinar, K., \& Brinkmann, S. 2008. Interviews: Learning the Craft of Qualitative Research Interviewing (Second ed.). London: Sage.

Ten Have, P. 2004. Understanding Qualitative Research and Ethnomethodology. London: Sage.

Wood, L., \& Zuber-Skerritt, O. 2013. PALAR as a methodology for community engagement?: A South African perspective. South African Journal of Education, 33(4).

Yin, R. K. 2013. Case Study Research: Design and Methods (Fifth ed.). London: Sage Publications.

Zuber-Skerritt, O. 1992a. Professional Development in Higher Education: A Theoretical Framework for Action Research. London: Kogan Page.

Zuber-Skerritt, O. 1992b. Action Research in Higher Education: Examples and Reflections (1996 Second ed.). London: Kogan Page.

Zuber-Skerritt, O. 2009. Action Learning and Action Research: Songlines Through Interviews. Rotterdam, The Netherlands: Sense Publishers.

Zuber-Skerritt, O. 2011. Action Leadership: Towards a Participatory Paradigm. Dordrecht, The Netherlands: Springer International.

Zuber-Skerritt, O., \& Teare, R. (2013). Lifelong Action Learning for Community Development: Learning and Development for a Better World. Rotterdam, The Netherlands: Sense Publishers.

Zuber-Skerritt, O., Fletcher, M., \& Kearney, J. (2014, in press). Professional Development in Higher Education and Communities: A New Vision for Action Research. Basingstoke UK: Palgrave Macmillan. 